\section{ANÁLISE CRÍTICA DA QUALIDADE DOS CENTROS DE PESQUISA CLÍNICA DE BELO HORIZONTE}

\author{
Karine Sampaio Lima \\ Bruna Bastos Vidal \\ Ana Paula Drummond Lage \\ Alberto Julius Alves Wainstein
}

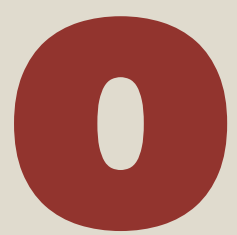

desenvolvimento de novos fármacosea pesquisa de novas tecnologias tem se tornado atividade estratégica, movimentando dezenas de bilhões de dólares e empregando centenas de milhares nas redes de saúde e tecnologia, com crescimento constante nas últimas décadas e acentuado impacto científico, econômico e estratégico mundial. Isso é ainda mais relevante quando aplicado ao câncer, onde a pesquisa clínica pode significar o acesso a drogas inovatórias. Não raro, os tratamentos padronizados e em uso para alguns tumores, tanto no sistema público como privado são baseados em drogas desenvolvidas há mais de 30 anos. Dezenas de novos conhecimentos e plataformas tecnológicas estão revolucionando os tratamentos oncológicos. O número de candidatos a produtos é tão extenso, que não apenas pacientes, mas mesmo profissionais de saúde ficam inseguros quanto às indicações e benefícios. Dessa maneira, fazem-se necessários, portanto, testes rigorosos para que seja garantida de maneira eficaz a segurança dos pacientes. Nesse contexto, a pesquisa clínica desempenha importante papel na avaliação dos processos. É pautada no princípio das boas práticas clínicas (Good Clinical Practice - GCP), que são um conjunto de regulamentações, diretrizes e padrões de qualidade ética e científica por meio dos quais os estudos envolvendo seres humanos devem ser desenhados, planejados, implementados, monitorados, auditados, analisados e concluídos. Os ensaios clínicos são sempre conduzidos de forma que os direitos e o bem-estar dos sujeitos da pesquisa, assim como a credibilidade dos dados dos ensaios clínicos, estejam sempre garantidos (1). É importante que os centros envolvidos em todos os países sigam os mesmos padrôes de segurança e qualidade de maneira a proteger e garantir a segurança do sujeito de pesquisa. Isso permite também assegurar que todos os dados e informações colhidos possam ser avaliados e validados de maneira uniforme.

De forma bem abrangente, definem-se os centros de pesquisa clínica como locais que exercem papel de grande relevância na condução dos estudos clínicos, sendo responsáveis pela administração, gestão e condução de estudos. A Resolução de Diretoria Colegiada (RDC) no 39 de 05 de junho de 2008 (2) define os centros de pesquisa como organizaçōes privadas ou públicas, legitimamente constituídas, nas quais são realizadas pesquisas clínicas. Um centro de pesquisa pode ou não estar inserido em um hospital ou em uma clínica médica. Para a Resolução, o termo "Instituição de Pesquisa" é utilizado como sinônimo de "Centro de Pesquisa”. Essas instituiçōes podem ser de pequeno, médio ou grande porte, vinculadas ou não a universidades e, em muitos casos, a hospitais universitários.
No Brasil, a pesquisa clínica passou a ter de fato normas regulamentadoras a partir de 1996, com a publicação da Resolução no 196 do Conselho Nacional de Saúde (CNS) (3), um marco na história da pesquisa clínica no nosso país, que incorpora os aspectos dos quatro princípios prima facie da pesquisa em seres humanos - autonomia, não maleficência, beneficência e justiça - e visa a assegurar direitos e deveres dos sujeitos de pesquisa. Essa Resolução baseia-se nos principais documentos internacionais que tratam da ética de pesquisa envolvendo seres humanos: Código de Nuremberg (1947), Declaração de Helsinque (1964 e demais versōes de 1975, 1983 e 1989) e Propostas de Diretrizes Éticas Internacionais para Pesquisas Biomédicas Envolvendo Seres Humanos (CIOMS/OMS 1982 e 1993).

Grande parte da pesquisa médica e biomédica do país se fazia em cerca de duas dezenas de instituições fortemente centradas nas regiōes Sul e Sudeste (4). Porém, houve significativo crescimento e contínua ascensão da implementação de novos centros de pesquisa e estudos em diversas áreas terapêuticas.

Devido ao grande progresso da pesquisa clínica nos últimos anos, foi criada, na década de 1990, a Conferência Internacional de Harmonização - International Conference on Harmonisation (ICH) (5) -, que fez com que principalmente a Comunidade Europeia, Estados Unidos e o Japão harmonizassem padrōes para as boas práticas na pesquisa clínica. Após a elaboração do Guia de Boas Práticas Clínicas, pelo ICH, os países das Américas que dele não faziam parte, incluindo o Brasil, elaboraram um documento análogo ao GCP-ICH, conhecido como "Boas práticas clínicas: documento das Américas" (6). Esse documento, assinado em 2005 pelos países da Organização Pan-Americana da Saúde (Opas), passou a ser aceito pelas legislações brasileiras e utilizado como padrão para atividades envolvendo a condução de pesquisa clínica em todo o país.

A normatização brasileira para estudos que envolvam seres humanos foi instituída pela Agência Nacional de Vigilância Sanitária (Anvisa), CNS e outras instâncias do governo. Atualmente no país, o fluxo do processo regulatório da pesquisa clínica envolve as seguintes instâncias regulatórias: Comitês de Ética em Pesquisa (CEP), Comissão Nacional de Ética em Pesquisa (Conep) e Anvisa. Esses órgãos asseguram a condução adequada dos estudos desenvolvidos no Brasil.

Com o objetivo de avaliar a condução de estudos clínicos, a Anvisa, os patrocinadores e agências regulatórias internacionais, como a FDA (agência que regula medicamentos e alimentos nos Estados Unidos) e a Agência Europeia de Medicamentos, (EMA), podem realizar auditorias. A auditoria é um exame cuidadoso e sistemático das atividades desenvolvidas nos locais onde são conduzidos os estudos clínicos cujos objetivos principais são averiguar se estes estão em conformidade com os padrōes éticos, determinar a validade e integridade dos dados e certificar a qualidade da condução dos estudos. As auditorias são comuns em casos de denúncias, suspeitas de fraude nos dados gerados, alto número de violações ao protocolo, notificação às agências regulatórias e suspeita de violação dos direitos dos sujeitos de pesquisa. Inspeções também podem ser realizadas pelos CEPs e Conep e buscam conformidade 
aos regulamentos, boas práticas clínicas, normas ou especificações e o subsequente relato de resultados.

Já os centros de pesquisa podem realizar autoavaliações, a fim de fornecer uma visão global do desempenho da organização e ajudar a identificar áreas que requeiram melhorias e determinar prioridades (7). Para a pesquisa clínica é fundamental que os estudos sejam desenvolvidos de forma a garantir a acurácia e qualidade dos dados, bem como sua integridade, confiabilidade e reprodutibilidade, garantindo a segurança dos sujeitos de pesquisa e a confiabilidade dos dados gerados (8).

Precisamente em 11 de maio de 2009, foi criada no Brasil, pela Anvisa, a Instrução Normativa no 4 (IN4) (9), com o propósito de obter melhorias no âmbito da pesquisa clínica no país. A IN4 sumariza um novo marco legal para adequação da condução de estudos clínicos ao grande desenvolvimento do mercado atual, para tentar garantir a qualidade dos estudos desenvolvidos, assim como seus resultados, preservando-se também a segurança dos sujeitos de pesquisa. Através desse roteiro, possíveis erros e carências podem ser diagnosticados a fim de corrigi-los ou adequá-los.

A uniformização dos padrões de qualidade relacionados à aprovação de estudos, operação, gestão, capacitação e desenvolvimento de estudos clínicos é muito importante para atingir excelência em pesquisa clínica. Isso também pode gerar visibilidade e novos investimentos para os centros de pesquisa, assim como a permeabilidade desse sistema e a capacidade de troca de informaçóes entre os pesquisadores, visando diretamente à eficiência do sistema como um todo.

PESQUISA CLÍNICA EM BELO HORIZONTE O presente estudo buscou diferentes tipos de instituições de pesquisa para avaliação regional fidedigna da qualidade dos centros de pesquisa clínica de Belo Horizonte. Trata-se de um estudo prospectivo do tipo transversal descritivo, onde foram abordadas instituições privadas e/ou públicas e/ou acadêmicas, envolvendo diferentes especialidades médicas.

Foram avaliados centros de pesquisa clínica com participação voluntária, mediante autorização das instituições envolvidas e assinatura do Termo de Consentimento Livre Esclarecido (TCLE).

Os critérios de elegibilidade para inclusão dos centros de pesquisa foram:

\section{Critérios de inclusão:}

- Centros de pesquisa clínica situados em Belo Horizonte que forneceram carta de autorização para coleta de dados.

- Centros de pesquisa clínica situados em Belo Horizonte que concordaram em assinar o TCLE e que responderam voluntariamente ao questionário do estudo até data predeterminada ou conclusão do estudo.

- Centros de pesquisa clínica em diversas áreas da saúde, sem restrição.

- Centros de pesquisa clínica com atuação no mercado e experiência mínima de três anos ou pelo menos experiência na condução de cinco estudos clínicos.
A avaliação foi realizada através do preenchimento de questionário autoaplicável, elaborado com base na IN4 da Anvisa, normas e regulamentações preestabelecidas para pesquisa clínica.

As questões aplicáveis à IN4 foram classificadas de acordo com o documento da Anvisa, como: críticas (C), maiores (M) e menores $(\mathrm{Me})$. As observações críticas são relacionadas diretamente à segurança do sujeito, podendo resultar em óbito, risco de morte ou condições inseguras e invalidação dos dados da pesquisa. As observaçóes maiores podem resultar em risco à saúde do sujeito de pesquisa ou invalidação dos dados. As observações menores indicam deficiência e/ou desvio e devem ser citadas para realização de melhorias na condução de estudos.

0 PERFIL DOS CENTROS DE ESTUDOS CLÍNICOS ANALISADOS Foram contatados 16 centros de pesquisa clínica de Belo Horizonte, sendo que, destes, sete foram incluídos no estudo, um não aceitou participar do estudo, cinco não retornaram aos contatos efetuados e três foram excluídos devido a não apresentarem os critérios de elegibilidade preestabelecidos.

Os centros avaliados dividiram-se entre instituiçôes privadas (4), públicas (1) e acadêmicas (2), de pequeno (3), médio (1) e grande porte (3). Porém, apesar das diferenças estruturais, logísticas e burocráticas entre essas instituições, não foram registradas diferenças significativas na avaliação dos questionários aplicados em cada uma.

As áreas terapêuticas, sob investigação, observadas com alto número de estudos foram oncologia (25\%) e infectologia (25\%), seguidas pela cardiologia, parasitologia, clínica médica, pneumologia, reumatologia e endocrinologia, com cerca de $8 \%$ cada.

Dos centros avaliados, os estudos clínicos dividiram-se principalmente entre fase II (39\%) e fase III $(58,4 \%)$. Grande parte dos patrocinadores investe em estudos de fase III no Brasil. Essa é uma tendência nacional que corresponde à tendência mundial, em que apenas quando se adquire excelência em estudos de fase III é que o país começa a ser considerado para estudos mais complexos como fase II e mesmo fase I.

Por se tratar de uma área de atuação com profissionalização e regulamentação recente, a pesquisa clínica vem se desenvolvendo nos últimos anos em Belo Horizonte e por esse motivo muitos centros de pesquisa estão em fase de maturação e não possuem ainda em seu histórico número representativo de estudos conduzidos. Porém, apurou-se que alguns desses centros estão em rápida ascensão, devido ao alto nível científico de seus investigadores e interesse na área. Entre os sete centros avaliados, foram identificados dois com 60 estudos cada (estudos abertos e fechados); dentre estes, um deles possuía 20 estudos em fase de recrutamento de sujeitos de pesquisa.

Entre os estudos com a inclusão de sujeitos de pesquisa aberta, ou seja, que demandam mais trabalho e esforço da equipe, constata-se alto número de estudos sendo conduzidos por cada coordenador e investigador principal, o que pode vir a sobrecarregar as atividades e prejudicar o desempenho do centro (Gráficos 1 e 2). O questionário de inspeção da IN4 aborda o número de estudos conduzidos por cada investigador principal, porém não especifica qual número é considerado adequado ou ideal. Acreditamos que a definição da 
capacidade ou número ideal de estudos por investigador principal e/ou coordenador de pesquisa dependa da complexidade e demanda de cada estudo.

O número de pesquisadores envolvidos ainda não é satisfatório para um crescimento mais significativo no setor. Nos sete centros avaliados, foram identificados apenas um a oito investigadores principais por centro.

Os pontos positivos identificados em todos os centros de pesquisa foram:

- Todos os comitês de ética em pesquisa envolvidos estão registrados na Conep;

- Conformidade com os princípios éticos vigentes na pesquisa clínica no atendimento aos sujeitos de pesquisa;

- Protocolos, documentos gerais e emendas foram previamente aprovados pelas agências regulatórias;

- Todos os documentos regulatórios e de condução dos estudos estão disponíveis para as agências regulatórias;

- Taxa anual de recrutamento geral de sujeitos de pesquisa nos centros avaliados está entre 0 e 20 pacientes para um centro, entre 21 e 50 pacientes para cinco centros e acima de 50 pacientes para outro centro. Esta taxa varia de acordo com a complexidade dos critérios de elegibilidade;

- Sujeitos de pesquisa possuem alta adesão aos procedimentos dos estudos;

- Equipe treinada em boas práticas clínicas, nos procedimentos dos estudos e procedimentos de emergência;

- Centros adequados ao armazenamento de medicamento com controle de temperatura, controle da validade e elaboração das documentaçōes para controle e dispensação do mesmo;

- Randomização dos sujeitos de pesquisa e condução do protocolo são seguidos de acordo com as instruções do patrocinador, bem como a adoção do método de estudo duplo-cego;

- Armazenamento e manipulação das amostras biológicas para os estudos são validados com controle de temperatura dos equipamentos e transporte adequados;

- Equipamentos utilizados para os estudos possuem registros de calibração periódica;

- Preenchimento correto das fichas clínicas ou Case Report Forms (CRF) de acordo com os documentos-fonte de cada paciente, com envio ao patrocinador no tempo previsto (24-72 horas);

- Centros estão de acordo com as normas para gerenciamento de resíduos coordenados pela própria estrutura hospitalar ou por serviço terceirizado.

Porém a avaliação dos dados obtidos da análise dos questionários revelou diversas não conformidades perante a IN4.

Em suma, considera-se o achado significativo, uma vez que, em um total de 66 questões classificáveis pela IN4, foram encontradas: três observaçōes menores, que correspondem a $3 / 4$ das questōes com esta classificação (75\%); seis maiores, que correspondem a $6 / 40$ (15\%); e quatro críticas (C), que correspondem a 4/19 (21,05\%).
De acordo com a IN4 da Anvisa (7), o não atendimento às observações maiores e críticas, e a consequente não conformidade no parecer final da Anvisa, poderão levar à conclusão de que o centro não apresenta nível satisfatório, de acordo com as boas práticas clínicas. $\mathrm{O}$ centro de pesquisa poderá ter o estudo em questão interrompido ou ter suspensas as atividades do investigador envolvido no estudo em questão ou mesmo o cancelamento definitivo da condução do protocolo clínico.

Apesar das questôes que abordam o controle da qualidade nos centros de pesquisa serem consideradas observações menores perante a Anvisa, o Sistema de Gestão de Qualidade (SGQ) nos centros é primordial. O SGQ aborda a elaboração, aplicação e treinamento em procedimentos operacionais padrão (POP); a estrutura organizacional do centro; as responsabilidades; os procedimentos, processos e recursos; a identificação e seleção de investigadores; o fluxo de aprovação regulatória; a elaboração adequada de TCLE; a capacitação de profissionais; as ferramentas para coleta de dados; as instruçôes sobre monitoria e auditoria; a condução e avaliação de resultados e o desenvolvimento de novas ferramentas.

Com o objetivo de avaliar os padrôes de qualidade para cada atividade exercida na condução dos estudos e buscar um padrão de excelência nos processos do centro de pesquisa, auto inspeções e auditorias externas ou internas devem ser realizadas, nos diversos setores, a fim de buscar melhorias para todo o sistema na condução dos estudos clínicos.

CONCLUSÕES Visto o interesse dos coordenadores e pesquisadores dos centros avaliados em se adequar às normas vigentes e expandir as atividades, apesar do achado significativo, considera-se que o cenário atual da pesquisa clínica em Belo Horizonte é promissor. Para uma gestão de excelência e padrão de qualidade satisfatório, é imperativo que os centros tomem as devidas providências para correção das não conformidades encontradas, de forma a se adequarem plenamente às agências regulatórias e não prejudicarem a condução dos estudos.

Fica evidente a importância da inserção de novas ferramentas e estratégias para melhor controle dos processos e da qualidade, uma vez que as atividades dos centros de pesquisa possuem importante, complexo e decisivo papel na condução dos estudos clínicos, principalmente no que se refere à segurança, proteção e atendimento dos sujeitos de pesquisa, boas práticas clínicas e aspectos regulatórios. É imprescindível manter a qualidade para gerar confiabilidade e acurácia dos resultados e agilidade nos procedimentos. A qualidade dos dados gerados em um estudo é uma exigência prioritária da pesquisa clínica para o uso e aplicação dessas informaçôes. O monitoramento de todos os processos, internos ou externos, bem como a formação profissional e a melhor capacitação evitam erros, desvios, violaçōes e questionamentos.

Avaliar a qualidade dos centros em seus diferentes aspectos contribui para a adequação e padronização de normas nacionais e internacionais e auxilia na preservação da credibilidade dos centros diante de agências regulatórias, agências de fomento, dos sujeitos de pesquisa e patrocinadores de estudos em geral. Nesse contexto, 


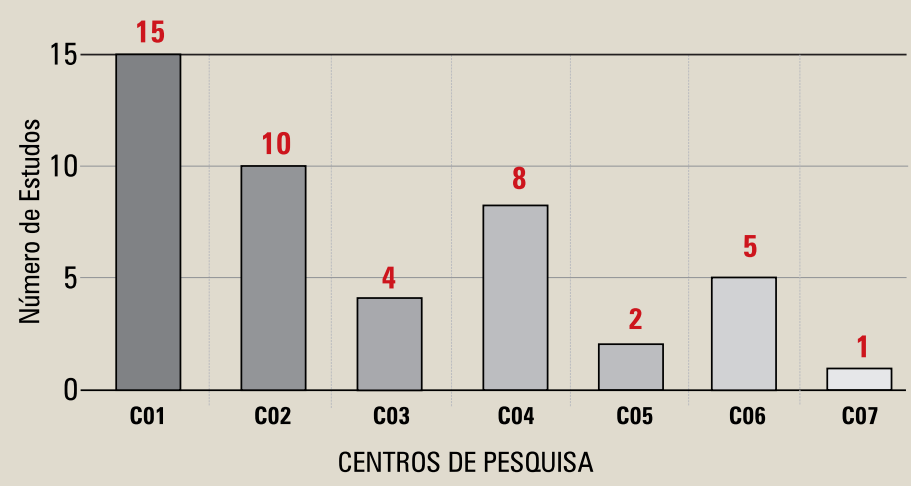

a utilização de um roteiro bem estruturado por parte de auditorias internas ou externas e inspeçôes facilita o processo de avaliação e permite discussão sobre o que deve ser revisto a fim de melhorar ou manter a qualidade.

As conclusões dessa pesquisa, bem como a análise dos questionários e achados desta investigação, foram disponibilizadas de maneira sigilosa a cada centro avaliado, com o intuito de contribuir de forma construtiva para o processo de detecção de não conformidades e aprimoramento da qualidade.

O processo de validação e registro de novo fármacos e biológicos é longo em todo o mundo e mais ainda no Brasil, onde além da eficácia e segurança, o custo é uma pendência considerada de acordo com a realidade do sistema de saúde brasileiro. Em muitas doenças, com grande premência no câncer, a maioria dos pacientes não tem tempo para esperar essas longas aprovaçōes. Neste contexto, os protocolos clínicos são uma ferramenta democrática para que pacientes com câncer, que não tem como se deslocar ao exterior, possam ter acesso no Brasil a inovaçóes que precisam como uma esperança imediata. Neste contexto, garantir a segurança e proteger esses pacientes é mister para um acesso ético e científico a essas inovações. Neste ambiente os pacientes e familiares têm acesso a informaçōes de maneira muito ágil e podem pressionar a equipe assistencial mesmo não conseguindo entender porque este acesso no Brasil é muito mais difícil do que em muitos outros países.

Espera-se, com esses resultados, relatar a nossa realidade na condução de estudos clínicos a partir do levantamento dos principais pontos deficientes e dificuldades em relação ao gerenciamento de estudos e controle de qualidade dos centros de pesquisa clínica em Belo Horizonte, Minas Gerais.

Karine Lima é biomédica, especialista em pesquisa clinica pela FCMMG, mestre em ciências da saúde pela Universidade Federal de Minas Gerais (UFMG).

Bruna Bastos é biomédica e especialista em pesquisa clinica pela Faculdade de Ciências Médicas de Minas Gerais (FCMMG).

Ana Paula Drummond Lage é farmacêutica, mestre e doutora em fisiologia pela UFMG, professora adjunta da FCMMG, diretora cientifica da Trymed Pesquisa Clinica. Email: anapdru@ gmail.com

Alberto Wainstein émédico, cirurgiāo oncológico da Oncad, mestre e doutor em medicina, professor adjunto da FCMMG, coordenador do serviço de oncologia da FHEMIG, presidente do Grupo Brasileiro de Melanoma.

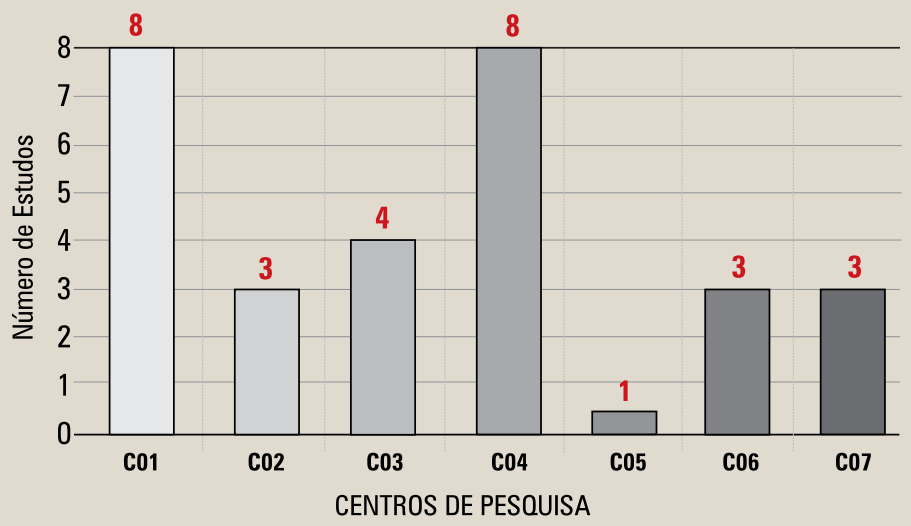

\section{REFERÊNCIAS BIBLIOGRÁFICAS}

1. European Medicines Agency (EMEA). About us.. Disponível em: http://www.ema.europa.eu/ema/index.jsp?curl=pages/about_us/ general/general_content_000235.jsp\&murl=menus/about_us/ about_us.jsp\&mid (acesso em 24/06/2011).

2. Brasil. Agência Nacional de Vigilância Sanitária (Anvisa). Resolução - RDC n० 39 de 05 de junho de 2008.

3. Brasil. Conselho Nacional de Saúde (CNS). Resolução - RDC n 196 de 10 de outubro de 1996.

4. Zago, M. A. "A pesquisa clínica no Brasil". Rio de Janeiro, Cienc Saúde Colet 9, 2, 2004.

5. International Conference on Harmonisation of Technical Requirements for Registration of Pharmaceuticals for Human Use. ICH Harmonised Tripartite Guideline for Good Clinical Practice E6(R1). 1996.

6. Brasil. Agência Nacional de Vigilância Sanitária (Anvisa).Documento das Américas. Organização Pan-Americana da Saúde. 2010.

7. Lima, J. S.; La Reza, D.; Teixeira, S.; Costa, C. "Pesquisa clínica: fundamentos, aspectos éticos e perspectivas". Rev Socerj 16, 225, 2003.

8. Associação Brasileira de Normas Técnicas (ABNT). NBR ISO 9000:2005 Sistemas de Gestão da Qualidade: fundamentos e vocabulário. ABNT 2006.

9. Brasil. Agência Nacional de Vigilância Sanitária (Anvisa). Instrução Normativa $n^{0}$ 4, de 11 de maio de 2009. 